\title{
Supersolvable Frobenius groups with nilpotent centralizers
}

\author{
Jhone Caldeira and Emerson de Melo
}

\begin{abstract}
Let $F H$ be a supersolvable Frobenius group with kernel $F$ and complement $H$. Suppose that a finite group $G$ admits $F H$ as a group of automorphisms in such a manner that $C_{G}(F)=1$ and $C_{G}(H)$ is nilpotent of class $c$. We show that $G$ is nilpotent of $(c,|F H|)$-bounded class.
\end{abstract}

\section{Introduction}

Let a group $A$ act by automorphisms on a group $G$. We denote by $C_{G}(A)$ the set $C_{G}(A)=\left\{x \in G ; x^{a}=x\right.$ for all $\left.a \in A\right\}$, the centralizer of $A$ in $G$ (the fixed-point subgroup). Very often the structure of $C_{G}(A)$ has strong influence over the structure of $G$. Recently, prompted by Mazurov's problem 17.72 in the Kourovka Notebook 8, some attention was given to the situation where a Frobenius group $F H$ acts by automorphisms on a finite group $G$. Recall that a Frobenius group $F H$ with kernel $F$ and complement $H$ can be characterized as a finite group that is a semidirect product of a normal subgroup $F$ by $H$ such that $C_{F}(h)=1$ for every $h \in H \backslash\{1\}$. By Thompson's theorem [11] the kernel $F$ is nilpotent, and by Higman's theorem [4] the nilpotency class of $F$ is bounded in terms of the least prime divisor of $|H|$.

In the case where the kernel $F$ acts fixed-point-freely on $G$, some results on the structure of $G$ were obtained by Khukhro, Makarenko and Shumyatsky in [7]. The authors prove that various properties of $G$ are in a certain sense close to the corresponding properties of its subgroup $C_{G}(H)$, possibly also depending on $H$. In particular, they proved the following result.

2010 Mathematics Subject Classification. Primary 20D45; secondary 17B70, 20D15, 20F40.

Key words and phrases. Frobenius groups of automorphisms; fixed-point-free; nilpotency class. 
Theorem 1.1 ([7], Theorem 2.7 (c)). Suppose that a finite group $G$ admits a Frobenius group of automorphisms $F H$ with kernel $F$ and complement $H$ such that $C_{G}(F)=1$ and $C_{G}(H)$ is nilpotent. Then $G$ is nilpotent.

Under the additional assumption that the Frobenius group of automorphisms $F H$ is metacyclic, that is, supposing that the kernel $F$ is cyclic, the authors use some Lie ring methods to obtain upper bounds for the nilpotency class of $G$. They proved that if $C_{G}(H)$ is nilpotent of class $c$, then the nilpotency class of $G$ is bounded in terms of $c$ and $|H|$. In the case when $G F$ is also a Frobenius group with kernel $G$ and complement $F$ (so that $G F H$ is a double Frobenius group) the latter result was obtained earlier in [10]. Examples in [7] show that the result on the nilpotency class of $G$ is no longer true in the case of non-metacyclic Frobenius groups.

Recall that a group $N$ is supersolvable if $N$ possesses a normal series with cyclic factors such that each term is normal in $N$. It is easy to see that a supersolvable group possesses a chief series whose factors have prime order.

Throughout the paper we use the expression " $(a, b, \ldots)$-bounded" to mean "bounded from above in terms of $a, b, \ldots$ only". In the present paper we consider the situation in which a not necessarily metacyclic Frobenius group acts by automorphisms on a finite group. More precisely, we prove the following theorem.

THEOREM 1.2. Let FH be a supersolvable Frobenius group with kernel $F$ and complement $H$. Suppose that $F H$ acts on a finite group $G$ in such a way that $C_{G}(F)=1$ and $C_{G}(H)$ is nilpotent of class $c$. Then $G$ is nilpotent of $(c,|F H|)$-bounded class.

Note that $G$ in Theorem 1.2 is nilpotent by Theorem 1.1, We wish to show that the nilpotency class of $G$ is $(c,|F H|)$-bounded. It should be mentioned that in the case of metacyclic $F H$, Khukhro, Makarenko and Shumyatsky in [7, Theorem 5.1] give a bound independent of the order $|F|$. At present it is unclear if in Theorem 1.2 the bound can be made independent of $|F|$. The proof is based on an analogous result on Lie algebras.

In [1, 6] we can find some other results bounding the nilpotency class for groups acted on by Frobenius groups of automorphisms with non-cyclic kernels. 


\section{Some results on graded Lie algebras}

Let $A$ be an additively written abelian group and $L$ an $A$-graded Lie algebra:

$$
L=\bigoplus_{a \in A} L_{a}, \quad\left[L_{a}, L_{b}\right] \subseteq L_{a+b}
$$

The particular case of $\mathbb{Z} / n \mathbb{Z}$-graded Lie algebras arises naturally in the study of Lie algebras admitting an automorphism $\varphi$ of order $n$. This is due to the fact that, after the ground field is extended by a primitive $n$th root of unity $\omega$, the eigenspaces $L_{i}=\left\{x \in L ; x^{\varphi}=\omega^{i} x\right\}$ behave like the components of a $\mathbb{Z} / n \mathbb{Z}$-grading. For example, the proof of a well-known theorem of Kreknin [9] stating that a Lie ring $L$ admitting a fixed-point-free automorphism of finite order $n$ is soluble with derived length bounded by a function of $n$, is reduced to proving the solvability of a $\mathbb{Z} / n \mathbb{Z}$-graded Lie ring with $L_{0}=0$.

THEOREM 2.1. Let $n$ be a positive integer and $L$ be a $\mathbb{Z} / n \mathbb{Z}$-graded Lie algebra. If $L_{0}=0$, then $L$ is solvable of $n$-bounded derived length.

In the special case where $n$ is a prime we have the following wellknown result proved by Higman.

THEOREM 2.2. [4] Let $p$ be a prime number and $L$ be $a \mathbb{Z} / p \mathbb{Z}$-graded Lie algebra. If $L_{0}=0$, then $L$ is nilpotent of p-bounded class.

If $J, Y, J_{1}, \ldots, J_{s}$ are subsets of $L$ we use $[J, Y]$ to denote the subspace of $L$ spanned by the set $\{[j, y] ; j \in J, y \in Y\}$ and for $i \geq 2$ we write $\left[J_{1}, \ldots, J_{i}\right]$ for $\left[\left[J_{1}, \ldots, J_{i-1}\right], J_{i}\right]$.

The next two results are also criteria for solvability and nilpotency of graded Lie algebra, respectively.

TheOREM 2.3. [5, Theorem 1] Let $n$ be a positive integer and $L$ be a $\mathbb{Z} / n \mathbb{Z}$-graded Lie algebra. Suppose that $[L, \underbrace{L_{0}, \ldots, L_{0}}_{m}]=0$. Then, $L$ is solvable of $(n, m)$-bounded derived length.

Theorem 2.4. [6, Proposition 2] Let $A$ be an additively written abelian group and $L$ an A-graded Lie algebra. Suppose that there are at most d nontrivial grading components among the $L_{a}$. If $[L, \underbrace{L_{a}, \ldots, L_{a}}_{m}]=$ 0 for all $a \in A$, then $L$ is nilpotent of $(d, m)$-bounded class. 
Now, let $p$ be a prime number and $L$ be a $\mathbb{Z} / p \mathbb{Z}$-graded Lie algebra. Assume that there exist non-negative integers $u$ and $v$ such that

$$
[L, \underbrace{L_{0}, \ldots, L_{0}}_{u}]=0
$$

and

$$
[[L, L] \cap L_{0}, \underbrace{L_{a}, \ldots, L_{a}}_{v}]=0 \text {, for all } a \in \mathbb{Z} / p \mathbb{Z} .
$$

We finish this section showing that conditions (1) and (2) together are sufficient to conclude that $L$ is nilpotent of $(p, u, v)$-bounded class. By Theorem 2.3, condition (11) implies that $L$ is solvable with $(p, u)$ bounded derived length. Thus, we can use induction on the derived length of $L$. If $L$ is abelian, there is nothing to prove. Assume that $L$ is metabelian. In this case, $[x, y, z]=[x, z, y]$, for every $x \in[L, L]$ and $y, z \in L$.

For each $b \in \mathbb{Z} / p \mathbb{Z}$ we denote $[L, L] \cap L_{b}$ by $L_{b}^{\prime}$. By Theorem 2.4 it is sufficient to prove that there exists a $(p, u, v)$-bounded number $t$ such that

$$
[L_{b}^{\prime}, \underbrace{L_{a}, \ldots, L_{a}}_{t}]=0, \text { for all } a, b \in \mathbb{Z} / p \mathbb{Z} .
$$

If $b=0$, this follows from (2) with $t=v$. Suppose that $b \neq 0$. If $a=0$, the commutator is zero from (1) with $t=u$. In the case where $a \neq 0$ we can find a positive integer $s<p$ such that $b+s a=0(\bmod p)$. Therefore, we have $[L_{b}^{\prime}, \underbrace{L_{a}, \ldots, L_{a}}_{v+s}] \subseteq[L_{0}^{\prime}, \underbrace{L_{a}, \ldots, L_{a}}_{v}]$. We know that the latter commutator is 0 by (2).

These previous ideas were applied in a similar way in [3] and [2].

\section{Bounding nilpotency class of Lie algebras}

In this section $F H$ denotes a finite supersolvable Frobenius group with kernel $F$ and complement $H$.

Let $R$ be an associative ring with unity. Assume that the characteristic of $R$ is coprime with $|F|$ and the additive group of $R$ is finite (or locally finite). Let $L$ be a Lie algebra over $R$. The main goal of this section is to prove the following theorem.

TheOREM 3.1. Suppose that FH acts by automorphisms on $L$ in such a way that $C_{L}(F)=0$ and $C_{L}(H)$ is nilpotent of class $c$. Then $L$ is nilpotent of $(c,|F H|)$-bounded class. 
If $F$ is cyclic, then $L$ is nilpotent of $(c,|H|)$-bounded class by $[\mathbf{7}$, Theorem 5.1]. Therefore without loss of generality we may assume that $F$ is not a cyclic group.

Let $Z$ be a subgroup of prime order $p$ of $Z(F)$ such that $Z \triangleleft F H$ and let $\varphi$ be a generator of $Z$.

Now, let $\omega$ be a primitive $p$ th root of unity. We extend the ground ring of $L$ by $\omega$ and denote by $\tilde{L}$ the algebra $L \otimes_{\mathbb{Z}} \mathbb{Z}[\omega]$. The action of $F H$ on $L$ extends naturally to $\tilde{L}$. Note that $C_{\tilde{L}}(F)=0$ and $C_{\tilde{L}}(H)$ is nilpotent of class $c$. Since $L$ and $\tilde{L}$ have the same nilpotency class, it is sufficient to bound the class of $\tilde{L}$. Hence, without loss of generality it will be assumed that the ground ring contains $\omega$ so that we will work with $L$ rather than $\tilde{L}$.

For each $i=0, \ldots, p-1$ we denote by $L_{i}$ the $\varphi$-eigenspace corresponding to eigenvalue $\omega^{i}$, that is, $L_{i}=\left\{x \in L ; x^{\varphi}=\omega^{i} x\right\}$. We have

$$
L=\bigoplus_{i=0}^{p-1} L_{i} \text { and }\left[L_{i}, L_{j}\right] \subseteq L_{i+j(\bmod p)} .
$$

Note that $L_{0}=C_{L}(Z)$. It is easy to see that $F H / Z$ acts on $L_{0}$ in such a manner that $C_{L_{0}}(F / Z)=0$. Thus, by induction on $|F H|$ we conclude that $L_{0}$ is nilpotent of $(c,|F H|)$-bounded class.

A proof of the next lemma can be found in [7, Lemma 2.4]. It will be useful to decompose the subalgebra $L_{0}$ into the fixed-points of the subgroups $H^{f}, f \in F$.

Lemma 3.2. Suppose that a finite group $N$ admits a Frobenius group of automorphisms $K B$ with kernel $K$ and complement $B$ such that $C_{N}(K)=1$. Then $N=\left\langle C_{N}(B)^{y} ; y \in K\right\rangle$.

In the above lemma we can write $N=\left\langle C_{N}\left(B^{y}\right) ; y \in K\right\rangle$, since $C_{N}(B)^{y}=C_{N}\left(B^{y}\right)$.

For any $f \in F$ we denote by $V_{f}$ the subalgebra $C_{L}\left(Z H^{f}\right) \leq L_{0}$. Note that $Z H^{f}$ is also a Frobenius group and that $Z H^{f}=Z H$ whenever $f \in Z$.

Lemma 3.3. We have $L_{0}=\sum_{f \in F} V_{f}$.

Proof. The subalgebra $L_{0}$ is $F H$-invariant and so it admits the natural action by the group $F H / Z$. Moreover, $F / Z$ acts fixed-pointfreely on $L_{0}$. Since the additive group of $L$ is finite, it is immediate from Lemma 3.2 that $L_{0}=\left\langle C_{L_{0}}\left(H^{\tilde{f}}\right) ; \tilde{f} \in F / Z\right\rangle$. As a consequence we have that $L_{0}=\left\langle C_{L}\left(Z H^{f}\right) ; f \in F\right\rangle$.

It is clear that any conjugated $H^{f}$ of $H$ can be considered as a Frobenius complement of $F H$. Now we describe the action $H^{f}$ on 
the homogeneous components $L_{i}$. Since $H$ is cyclic, we can choose a generator $h$ of $H$ and $r \in\{1,2, \ldots, p-1\}$ such that $\varphi^{h^{-1}}=\varphi^{r}$. Then $r$ is a primitive $q$ th root of 1 in $\mathbb{Z} / p \mathbb{Z}$. The group $H$ permutes the homogeneous components $L_{i}$ as follows: $L_{i}^{h}=L_{r i}$ for all $i \in \mathbb{Z} / p \mathbb{Z}$. Indeed, if $x_{i} \in L_{i}$, then $\left(x_{i}^{h}\right)^{\varphi}=x_{i}^{h \varphi h^{-1} h}=\left(x_{i}^{\varphi^{r}}\right)^{h}=\omega^{r i} x_{i}^{h}$. On the other hand, since $F$ commutes with $\varphi$, we also have $L_{i}^{h^{f}}=L_{r i}$ for all $i \in \mathbb{Z} / p \mathbb{Z}$ (because if $x_{i} \in L_{i}$, then $\left(x_{i}^{h^{f}}\right)^{\varphi}=\omega^{r i} x_{i}^{h^{f}}$ ). Thus, the action of $H^{f}$ on the components $L_{i}$ coincides with the action of $H$.

To lighten the notation we establish the following convention.

REMARK 3.4 (Index Convention). In what follows, for a given $u_{s} \in$ $L_{s}$ we denote both $u_{s}^{h^{i}}$ and $u_{s}^{\left(h^{f}\right)^{i}}$ by $u_{r^{i} s}$, since $L_{s}^{h^{i}}=L_{s}^{\left(h^{f}\right)^{i}}=L_{r^{i} s}$. Therefore, we can write $u_{s}+u_{r s}+\cdots+u_{r^{q-1} s}$ to mean a fixed-point of $H^{f}$ for any $f \in F$.

LEMma 3.5. There exists a $(c,|F H|)$-bounded number $u$ such that $[L, \underbrace{L_{0}, \ldots, L_{0}}_{u}]=0$.

Proof. It suffices to prove that $[L_{b}, \underbrace{L_{0}, \ldots, L_{0}}_{u}]=0$ for any $b \in$ $\{0,1, \ldots, p-1\}$.

Taking into account that $L_{0}$ is nilpotent of $(c,|F H|)$-bounded class, we may assume $b \neq 0$.

By Lemma 3.3, $L_{0}=\sum_{f} V_{f}$. Let $u_{b} \in L_{b}$. Since $u_{b}+u_{r b}+\cdots+$ $u_{r^{q-1} b} \in C_{L}\left(H^{f}\right)$ and $V_{f} \subseteq C_{L}\left(H^{f}\right)$, we have

$$
[u_{b}+u_{r b}+\cdots+u_{r^{q-1}}, \underbrace{V_{f}, \ldots, V_{f}}_{c}]=0 .
$$

Thus, $\sum_{i=0}^{q-1}[u_{r^{i} b}, \underbrace{V_{f}, \ldots, V_{f}}_{c}]=0$. On the other hand, $[u_{r^{i} b}, \underbrace{V_{f}, \ldots, V_{f}}_{c}] \in$ $L_{r^{i} b}$ and $L_{r^{i} b} \neq L_{r^{j} b}$ whenever $i \neq j$. Therefore, we obtain $[L_{b}, \underbrace{V_{f}^{c}, \ldots, V_{f}}_{c}]=$ 0 .

Let $S$ be an $F H$-invariant subalgebra of $L_{0}$ and let $S_{f}=S \cap V_{f}$ for $f \in F$. It follows that $S=\sum_{f} S_{f}$. Now, we will show that there exists a $(c,|F H|)$-bounded number $u$ such that $[L_{b}, \underbrace{S, \ldots, S}_{u}]=0$ using induction on the nilpotency class of $S$. Since $[S, S]$ is nilpotent of smaller class, there exists a $(c,|F H|)$-bounded number $u_{1}$ such that $[L_{b}, \underbrace{[S, S], \ldots,[S, S]}_{u_{1}}]=0$. 
Now, set $l=(c-1)|F|+1$ and $W=\left[L_{b}, S_{i_{1}}, \ldots, S_{i_{l}}\right]$ for some choice of $S_{i_{j}}$ in $\left\{S_{f} ; f \in F\right\}$. It is clear that for any permutation $\pi$ of the symbols $i_{1}, \ldots, i_{l}$ we have $W \leq\left[L_{b}, S_{\pi\left(i_{1}\right)}, \ldots, S_{\pi\left(i_{l}\right)}\right]+\left[L_{b},[S, S]\right]$. Also, note that the number $l$ is big enough to ensure that some $S_{i}$ occurs in the list $S_{i_{1}}, \ldots, S_{i_{l}}$ at least $c$ times. Thus, we deduce that $W \leq$ $\left[[L_{b}, \underbrace{S_{i}, \ldots, S_{i}}_{c}], *, \ldots, *\right]+\left[L_{b},[S, S]\right]$, where the asterisks denote some of the subalgebras $S_{j}$ which, in view of the fact that $[L_{b}, \underbrace{S_{i}, \ldots, S_{i}}_{c}]=0$, are of no consequence. Hence, $W \leq\left[L_{b},[S, S]\right]$.

Further, for any choice of $S_{i_{1}}, \ldots, S_{i_{l}} \in\left\{S_{f} ; f \in F\right\}$ the same argument shows that

$$
\left[W, S_{i_{1}}, \ldots, S_{i_{l}}\right] \leq[W,[S, S]] \leq\left[L_{b},[S, S],[S, S]\right]
$$

More generally, for any $m$ and any $S_{i_{1}}, \ldots, S_{i_{m l}} \in\left\{S_{f} ; f \in F\right\}$ we have

$$
\left[L_{b}, S_{i_{1}}, \ldots, S_{i_{m l}}\right] \leq[L_{b}, \underbrace{[S, S], \ldots,[S, S]}_{m}] .
$$

Put $u=u_{1} l$. The above shows that

$$
\left[L_{b}, S_{i_{1}}, \ldots, S_{i_{u}}\right] \leq[L_{b}, \underbrace{[S, S], \ldots,[S, S]}_{u_{1}}]=0 .
$$

Of course, this implies that $[L_{b}, \underbrace{S, \ldots, S}_{u}]=0$. The lemma is now straightforward from the case where $\stackrel{u}{S}=L_{0}$.

\section{Proof of Theorem 3.1}

In view of Lemma 3.5, applying the arguments of Section 2, Theorem 3.1 holds if we show the following:

Lemma 3.6. Let $L$ be metabelian. There exists a $(c,|F H|)$-bounded number $v$ such that $[[L, L] \cap L_{0}, \underbrace{L_{a}, \ldots, L_{a}}_{v}]=0$, for all $a \in \mathbb{Z} / p \mathbb{Z}$.

Proof. Recall that $[L, L] \cap L_{0}=\sum_{f}[L, L] \cap V_{f}$ where $V_{f}=C_{L}\left(Z H^{f}\right)$. Set $V=C_{L}(Z H)$ and $V^{\prime}=[L, L] \cap V$. First we prove that $[V^{\prime}, \underbrace{L_{a}, \ldots, L_{a}}_{v}]=$ 0 for any $a \in \mathbb{Z} / p \mathbb{Z}$.

For any $u_{a} \in L_{a}$ we have $u_{a}+u_{r a}+\cdots+u_{r^{q-1} a} \in C_{L}(H)$ (under Index Convention). Thus,

$$
[V^{\prime}, \underbrace{u_{a}+u_{r a}+\cdots+u_{r^{q-1} a}, \ldots, v_{a}+v_{r a}+\cdots+v_{r^{q-1} a}}_{c}]=0
$$


for any $c$ elements $u_{a}, \ldots, v_{a} \in L_{a}$.

Let $T$ denote the span of all the sums $x_{a}+x_{r a}+\cdots+x_{r^{q-1} a}$ over $x_{a} \in L_{a}$ (in fact, $T$ is the fixed-point subspace of $H$ on $\bigoplus_{i=0}^{q-1} L_{r^{i} a}$ ). Then the latter equality means that

$$
[V^{\prime}, \underbrace{T, \ldots, T}_{c}]=0 .
$$

Applying $\varphi^{j}$ we also obtain

$$
[V^{\prime}, \underbrace{T^{\varphi^{j}}, \ldots, T^{\varphi^{j}}}_{c}]=[\left(V^{\prime}\right)^{\varphi^{j}}, \underbrace{T^{\varphi^{j}}, \ldots, T^{\varphi^{j}}}_{c}]=0 .
$$

A Vandermonde-type linear algebra argument shows that $L_{a} \subseteq$ $\sum_{j=0}^{q-1} T^{\varphi^{j}}$. Actually this fact is a consequence of the following result:

LEMma 3.7. [7, Lemma 5.3] Let $\langle\alpha\rangle$ be a cyclic group of order $n$, and $\omega$ a primitive nth root of unity. Suppose that $M$ is a $\mathbb{Z}[\omega]\langle\alpha\rangle$ module such that $M=\sum_{i=1}^{m} M_{t_{i}}$, where $x \alpha=\omega^{t_{i}} x$ for $x \in M_{t_{i}}$ and $0 \leq t_{1}<t_{2}<\cdots<t_{m}<n$. If $z=y_{t_{1}}+y_{t_{2}}+\cdots+y_{t_{m}}$ for $y_{t_{i}} \in M_{t_{i}}$, then for some m-bounded number $d_{0}$ every element $n^{d_{0}} y_{t_{s}}$ is a $\mathbb{Z}[\omega]$-linear combination of the elements $z, z \alpha, \ldots, z \alpha^{m-1}$.

Now we can apply Lemma 3.7 with $\alpha=\varphi, M=L_{a}+L_{r a}+$ $\cdots+L_{r^{q-1} a}$ and $m=q$ to $w=u_{a}+u_{r a}+\cdots+u_{r^{q-1} a} \in T$ for any $u_{a} \in L_{a}$, because here the indices $r^{i} a$ can be regarded as pairwise distinct residues modulo $p$ ( $r$ is a primitive $q$ th root of 1 modulo $p$ ). Since $p$ is invertible in our ground ring, follows that $L_{a} \subseteq \sum_{j=0}^{q-1} T^{\varphi^{j}}$.

Set $v=(c-1) q+1$. We now claim that $[V^{\prime}, \underbrace{L_{a}, \ldots, L_{a}}_{v}]=0$. Indeed, after replacing $L_{a}$ with $\sum_{j=0}^{q-1} T^{\varphi^{j}}$ and expanding the sums, in each commutator of the resulting linear combination we can freely permute the entries $T^{\varphi^{j}}$, since $L$ is metabelian. Since there are sufficiently many of them, we can place at least $c$ of the same $T^{\varphi^{j_{0}}}$ for some $j_{0}$ right after $V^{\prime}$ at the beginning, which gives 0 .

Note that in the case where $f \in F \backslash Z$ we can consider the Frobenius group $Z H^{f}$ and conclude in a similar way that

$$
[[L, L] \cap V_{f}, \underbrace{u_{a}, \ldots, v_{a}}_{v}]=0
$$

for any $v$ elements $u_{a}, \ldots, v_{a} \in L_{a}$. 


\section{Proof of Theorem 1.2}

We will use the following results.

LEMMA 4.1. Let $G$ be a finite p-group admitting a nilpotent group of automorphisms $F$ such that $C_{G}(F)=1$. Let $F_{p^{\prime}}$ be the Hall $p^{\prime}$-subgroup of $F$. Then $C_{G}\left(F_{p^{\prime}}\right)=1$.

Proof. The subgroup $C_{G}\left(F_{p^{\prime}}\right)$ is $F$-invariant, and so, it admits the natural action by the $p$-group $F / F_{p^{\prime}}$. Since a finite $p$-group cannot act without nontrivial fixed points on another $p$-group, we must have $C_{G}\left(F_{p^{\prime}}\right)=1$.

LEMMA 4.2. [7, Lemma 2.2] Let $G$ be a finite group admitting a nilpotent group of automorphisms $F$ such that $C_{G}(F)=1$. If $N$ is an $F$-invariant normal subgroup of $G$, then $C_{G / N}(F)=1$.

Lemma 4.3. [7, Theorem 2.3] Suppose that a finite group $G$ admits a Frobenius group of automorphisms $F H$ with kernel $F$ and complement $H$. If $N$ is an $F H$-invariant normal subgroup of $G$ such that $C_{N}(F)=$ 1 , then $C_{G / N}(H)=C_{G}(H) N / N$.

We know that $G$ in Theorem 1.2 is nilpotent. We wish to show that the nilpotency class of $G$ is $(c,|F H|)$-bounded. It is easy to see that without loss of generality we may assume that $G$ is a $p$-group. Moreover, by Lemma 4.1 we also may assume that $(|G|,|F|)=1$.

Consider the associated Lie ring of the group $G$

$$
L(G)=\bigoplus_{i=1}^{n} \gamma_{i} / \gamma_{i+1},
$$

where $n$ is the nilpotency class of $G$ and $\gamma_{i}$ are the terms of the lower central series of $G$. The nilpotency class of $G$ coincides with the nilpotency class of $L(G)$. The action of the group $F H$ on $G$ induces naturally an action of $F H$ on $L(G)$. Since $F$ acts fixed-point-freely on every quotient $\gamma_{i} / \gamma_{i+1}$, it follows by Lemma 4.2 that $C_{L(G)}(F)=0$. We observe that the subring $C_{L(G)}(H)$ is nilpotent of class at most $c$ by Lemma 4.3 . Theorem 3.1 now tells us that $L(G)$ is nilpotent of $(c,|F H|)$-bounded class. The proof is complete.

\section{References}

[1] J. Caldeira, E. de Melo, P. Shumyatsky, On groups and Lie algebras admitting a Frobenius group of automorphisms, Journal of Pure and Applied Algebra, 216 (2012), 2730-2736.

[2] E. de Melo, J. Caldeira, On finite groups admitting automorphisms with nilpotent centralizers. J. Algebra To appear. 
[3] E. de Melo, P. Shumyatsky, Finite groups and their coprime automorphisms, Proc. Amer. Math. Soc., 145 (2017), 3755-3760.

[4] G. Higman, Groups and rings which have automorphisms without non-trivial fixed elements, J. London Math. Soc, 32 (1957), 321-334.

[5] E. I. Khukhro. On solvability of Lie rings with an automorphism of finite order, Siberian. Math. J., 42 (2001), 996-1000.

[6] E. I. Khukhro, P. Shumyatsky, Nilpotency of finite groups with Frobenius groups of automorphisms, Monatsh. Math., 163 (2011), 461-470.

[7] E. I. Khukhro, N. Y. Makarenko, P. Shumyatsky, Frobenius groups of automorphisms and their fixed points, Forum Math, 26 (2014), 73-112.

[8] E. I. Khukhro, V. D. Mazurov (Eds), Unsolved Problems in Group Theory. The Kourovka Notebook, 18th edition, Institute of Mathematics, Novosibirsk, (2014).

[9] V. A. Kreknin, The solubility of Lie algebras with regular automorphisms of finite period, Math. USSR Doklady, 4 (1963), 355-358.

[10] N.Y. Makarenko, P. Shumyatsky, Frobenius groups as groups of automorphisms, Proc. Amer. Math. Soc., 138 (2010), 3425-3436.

[11] J. G. Thompson, Finite groups with fixed-point-free automorphisms of prime order, Proc. Nat. Acad. Sci. USA 45 (1959), 578-581.

Instituto de Matemática e Estatística, Universidade Federal de GoiÁs, Goî̂nia-GO, 74690-900 BraziL

E-mail address: jhone@ufg.br

Department of Mathematics, Universidade de Brasília, BrasíliaDF, 70910-900 BRAZIL

E-mail address: emerson@mat.unb.br 